\title{
COMPETENCIAS SOCIOEMOCIONALES EN EL DESARROLLO PROFESIONAL DEL MAESTRO.
}

\section{(SOCIO-EMOTIONAL COMPETENCIES OF TEACHER PROFESSIONAL DEVELOPMENT)}

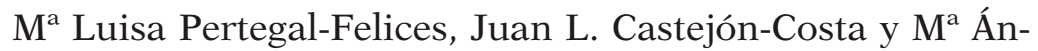
geles Martínez

Universidad de Alicante

\section{RESUMEN}

El principal objetivo de las universidades es educar en términos de adquisición de capacidades, habilidades, competencias y valores, con el fin último de promover el empleo.Numerosos autores han llegado a la conclusión de que la inteligencia emocional forma parte de las competencias que requieren las personas para desarrollar con éxito su labor profesional. En este trabajo se realiza una comparación de perfiles de competencias socioemocionales, mediante un análisis multivariado de la varianza, para el cual se dispuso de la opinión de una muestra de 148 maestros en ejercicio y de la medida en dichas competencias de 139 estudiantes de magisterio. Los resultados indicaron que existen diferencias en los perfiles de ambos grupos; siendo esta diferencia significativa para 11 de las 13 variables socioemocionales analizadas. Los estudiantes tienen menos desarrolladas las competencias socioemocionales que requieren según los profesionales. Parece, por tanto, necesario que desde los currícula universitarios se promueva el desarrollo de estas competencias para un desarrollo profesional eficaz.

\section{ABSTRACT}

The main purpose of universities is to educate in terms of knowledge, abilities, skills and values adquisition, with the ultimate aim of promoting employment. Many academics have concluded that intelligence is part of the social-emotional skills needed by individuals to successfully develop their professional work. In this article we undertake an analysis of socio-emotional 
competence profiles by means of the application of a multivariate procedure to a sample of 148 teachers in exercise and to 139 students in teacher training courses. The results of our analysis show notable differences in the profiles of both groups, the difference being highly significant in 11 of the 13 socioemotional variables analyzed. Students show a minor development of socioemotional skills required by professionals. As a main conclusion of our analysis we can state that it reveals imperative that the university curricula promotes the development of these skills in order to achieve an effective professional development.

\section{INTRODUCCIÓN}

Son muchos los estudios y organizaciones que incorporan, trabajan e investigan acerca de las emociones y su importancia en muchos aspectos de la vida del sujeto. En el ámbito educativo la UNESCO (1996), en su Informe Delors, destaca el papel de las emociones y hace hincapié en la necesidad de educar la dimensión emocional del ser humano junto a su dimensión cognitiva. La Declaración de Bolonia (Ministros de Educación de la Unión Europea, 1999) -que recoge los planteamientos para la creación de un espacio europeo común de enseñanza superior antes de 2010-, destaca la importancia de la educación en términos de adquisición, por parte del estudiante, de capacidades, habilidades, competencias y valores, adoptando una nueva metodología orientada al aprendizaje de competencias, entre ellas las socioemocionales; y el Proyecto Tuning Educational Structures in Europe (González y Wagenaar, 2003) -financiado por la Comisión Europea en el marco del Programa Sócrates- desarrolla perfiles profesionales, resultados del aprendizaje y competencias deseables en términos de competencias genéricas y relativas a cada área de estudios. Algunas de estas competencias guardan una estrecha relación con la inteligencia emocional y la personalidad. Están referidas a capacidades individuales relativas a la capacidad de expresar los propios sentimientos, habilidades críticas y de autocrítica; y destrezas sociales relacionadas con las habilidades interpersonales, la capacidad de trabajar en equipo o la expresión de compromiso social o ético.

Para describir a las personas emocionalmente inteligentes se han establecido diversos modelos. Así, los autores que parten desde un modelo de habilidades (Mayer y Salovey, 1997; Mayer, Caruso y Salovey 2000; Mayer, Salovey y Caruso, 2000) contemplan la inteligencia emocional (IE) como un conjunto de habilidades cognitivas para usar y manejar adaptativamente las emociones. Otros autores prefieren una perspectiva de la Inteligencia Emocional (IE) como rasgo, en la cual se incluyen algunos rasgos de personalidad claves para desarrollar comportamientos emocionalmente inteligentes (Bar-On, 2000; Cherniss, 2000; Goleman, 2001). 
Dentro del modelo de IE basado en habilidades se han elaborado diferentes instrumentos -tanto de autoinforme como de capacidad- que permiten su evaluación: la Trait Meta-Mood Scale-48 (TMMS-48), desarrollada por el grupo de investigación de Mayer y Salovey; la Spanish modified Trait MetaMood Scale-24 (TMMS-24), versión reducida de la TMMS-48 y adaptada al castellano por el grupo de investigación de Málaga (Fernández-Berrocal, Extremera y Ramos, 2004); el Schutte Self Report Inventory (SSRI) de Schutte, Malouff, Hall, Haggerty, Cooper, Golden y Dornheim (1998) -todas ellas medidas de autoinforme con una consistencia interna, fiabilidad y validez aceptables- y el Multifactor Emotional Intelligence Scale (MEIS) (Mayer, Caruso y Salovey, 1999) y el Mayer-Salovey-Caruso Emotional Intelligence Test (MSCEIT) (Mayer, Caruso y Salovey, 2002) basadas en un acercamiento práctico.

Para evaluar la IE desde los modelos mixtos se han creado diversos instrumentos -basados en medidas de autoinforme- destacando el Bar-On Emotional Quotient Inventory (EQ-i; Bar-On, 1997) adaptada al castellano por MHS; el Trait Emotional Intelligence Questionaire (TEIQue), creado por el grupo británico de Petrides y Furnham, y similar al de Bar-On (Petrides y Furnham, 2003) y el Emotional Competence Inventory (ECI de Goleman, 1998) construido para predecir la efectividad y el rendimiento personal en el mundo laboral y empresarial.

La búsqueda del máximo rendimiento de los trabajadores en el ámbito laboral, ha llevado a investigar las habilidades que poseen los empleados más exitosos que aumentan el rendimiento en la empresa. En el análisis de estas capacidades diversos autores (Boyatzis et al 2000; Goleman, 1995, 1998, 2001; Mayer y Salovey, 1990, 1997; Salovey y Mayer, 1990) han llegado a la conclusión de que la inteligencia no sólo general, también la socioemocional y los factores de personalidad forman parte del complejo entramado de competencias que requieren las personas para desarrollar con éxito su labor profesional. La relación entre las competencias emocionales y el rendimiento ha sido avalada por numerosas investigaciones (Boyatzis, 2006, 2008; Brotheridge y Lee, 2008; Cooper, 1997; Dreyfus, 2008; Koman y Wolff, 2008; Murga y Ortego, 2003).

Son muchos los estudios que muestran que la capacidad de adaptación de una persona al medio podría estar determinada por la inteligencia emocional (Boyatzis, Goleman y Rhee, 2000; Ciarrochi, Chan, y Caputi, 2000). Una buena adaptación podría ser la causa del éxito laboral en diversos ámbitos. Muestra de ello son los estudios realizados por Møller y Powell (2001), Rozeil, Pettijohn y Parker, (2001) y Sjöberg, (2001) en el ámbito laboral; Culver y Yokomoto (1999), Lam (1998) y Parker, (2002) en el terreno educativo; y Ciarrochi, Deane y Anderson (2002), Parker, Taylor y Bagby (2001) y Sa- 
lovey, (2001) en el de salud mental. Por otra parte, una mala adaptación podría tener consecuencias laborales negativas, tal es el caso del conocido síndrome de estar quemado o burnout (Aluja, Blanch, Biscarri, 2002). Otros estudios que analizan como el buen control del estrés o de otras variables emocionales evitan o reducen los estados de estrés o depresión en el trabajo, lo relacionan con estas variables en distintos ámbitos profesionales como son el sanitario (Aiken et al. 2002; Gil-Monte, 1991) y el educativo (Durán, Montalbán, Rey, Extremera, 2005).

La noción de competencia profesional pretende mejorar la relación del sistema educativo con el productivo, con el objetivo de impulsar una adecuada formación de los profesionales. Sin embargo, no existe un acuerdo unánime entre los expertos sobre el constructo de competencia emocional. El primer punto de discrepancia aparece en la propia designación. Así, mientras que algunos autores se refieren a la competencia emocional, otros prefieren utilizar la designación competencia socio-emocional; otros optan por utilizan el plural: competencias emocionales o socio-emocionales (Bisquerra y Pérez, 2007).

Existe una amplia variedad de definiciones y clasificaciones de competencia. Aunque el constructo está en debate, sí hay acuerdo en la importancia del desarrollo de las competencias emocionales (Bisquerra y Pérez, 2007). En el ámbito profesional, el término se basa en la capacidad, aptitud, habilidad, destreza o eficiencia que llevan a la persona a un desempeño exitoso en el trabajo (Boyatzis, 1982; González y Wagenaar, 2003; McClelland, 1973; OCDE, 2002; OIT, 2008). Para Cano (2005), hablar de competencias supone referirse a la capacidad del sujeto para movilizar los recursos que ha adquirido: los conocimientos, las habilidades y las actitudes, para afrontar y resolver una situación problemática.

En cuanto a la clasificación, se puede realizar de diversas formas atendiendo al eje sobre el que se establecen. La OCDE (2002) considera que la selección de las competencias clave depende de lo que las sociedades valoren, en cada momento y en cada contexto. En la actualidad, algunas de las propuestas más relevantes son las que proponen organismos como OIT/CINTERFOR (2008), la OCDE (2002) o González y Wagenaar (2003) en el Proyecto Tuning.

Los datos procedentes de las encuestas de inserción laboral destacan la importancia de incrementar la competencia en informática, en idiomas y en habilidades sociales al tiempo que proponen estimular la creatividad, la gestión, las competencias instrumentales, la toma de decisiones y el liderazgo (ANECA, 2005). 
En el ámbito profesional, recientemente se ha comenzado a analizar la relación entre la IE y el ajuste personal y bienestar del docente (Palomera, Fernández-Berrocal y Brackett, 2008). Aunque en España no existe tradición de investigación sobre estas competencias, en países anglosajones, entre los que destaca Estados Unidos, pueden encontrarse importantes y sólidas investigaciones a este respecto, especialmente desde la última década (Repetto y Pérez-González, 2007). Prueba de ello es que Estados Unidos gasta alrededor de 50 billones de dólares cada año en formación, de la cual, gran parte se centra en capacidades sociales y emocionales (Cherniss, 2000). Otros estudios que analizan la relación entre inteligencia emocional y burnout en profesores de secundaria (Chan, 2006), muestran cómo el burnout influye negativamente sobre el rendimiento de los alumnos y la calidad de su enseñanza; así como sobre el bienestar docente (Vanderberghe y Huberman, 1999) y afecta negativamente a las relaciones interpersonales profesor-alumno (Yoon, 2002).

En cuanto a las investigaciones dirigidas al profesorado podemos citar la que realizan Jennings y Greenberg (2009) y Sutton y Wheatly (2003) que ponen de manifiesto la estrecha relación entre las competencias sociales y emocionales de los profesores y la efectividad y calidad a la hora de llevar a cabo los procesos de enseñanza-aprendizaje del aula, así como el desarrollo de la conducta prosocial de los alumnos en clase. Di Fabio y Pazazzeschi (2008) evalúan la relación entre la inteligencia emocional y la autoeficacia en una muestra de profesores italianos. Un estudio similar es realizado por Chan (2008), quien estudia las relaciones entre inteligencia emocional, la autoeficacia y las habilidades de afrontamientoen profesores de Hong-Kong. En España cabe destacar el estudio realizado sobre la inteligencia emocional percibida y la satisfacción con la vida en profesores universitarios por Landa, López-Zafra, Martínez de Antonana y Pulido (2006).

A pesar de la importancia que se le concede a la inteligencia emocional para el desarrollo de la actividad profesional del profesorado son escasos los programas dirigidos a la formación del profesorado. En nuestro ámbito son varios los investigadores que han señalado la necesidad de desarrollar la inteligencia emocional del profesorado desde la formación inicial, como parte de las competencias genéricas establecidas por el EEES (Bisquerra, 2005; Bueno, Teruel y Valero, 2005; Extremera y Fernández-Berrocal, 2004; Pesquero, Sánchez, González y Martín, 2008; Sala y Abarca, 2002; Teruel, 2000; Vivas, 2004). Sin embargo, falta por establecer propuestas concretas acerca de cómo incluir estas competencias en el curriculum del profesorado, a pesar de que en algunos casos se ha puesto claramente en evidencia que el entrenamiento en competencias emocionales de los profesores noveles ha mostrado su efectividad no sólo en el aumento de su propia competencia 
emocional, sino también en la predicción de una transición ajustada del rol de estudiante a la vida profesional (Byron, 2001).

La misión que persiguen las universidades consiste, entre otras, en promover la obtención de empleo. El papel central de éstas en el Espacio Europeo de Educación Superior es el de educar en términos de adquisición, por parte del estudiante, de capacidades, habilidades, competencias y valores, adoptando una nueva metodología orientada al aprendizaje de competencias, entre las que se encuentran las socio-emocionales (Ministros de Educación de la Unión Europea, 1999). Weare y Grey (2003), en un estudio realizado sobre el desarrollo de competencias, concluyen con la recomendación de desarrollar explícitamente las competencias, tanto sociales como emocionales no sólo en la escuela, sino también en las instituciones dirigidas a la formación del profesorado, partiendo de la idea de que no es posible enseñar una competencia que previamente no se ha alcanzado, al igual que no es posible enseñar con calidad ante la ausencia de bienestar docente

La formación basada en competencias requiere la definición previa de las competencias necesarias para un adecuado desempeño profesional (Bisquerra y Pérez, 2007). Gil (2007) recoge cuatro fases en la evaluación de la selección basada en competencias: 1) definir los criterios de desempeño en el puesto a cubrir, 2) identificar las competencias personales que contribuirían a alcanzar los criterios de desempeño, 3) recoger información sobre las competencias que poseen los candidatos a través de técnicas como los tests, cuestionarios, entrevistas o ejercicios prácticos y 4) comparar las competencias valoradas en los aspirantes y las competencias unidas al puesto de trabajo para determinar el grado de ajuste de cada candidato al puesto.

El objetivo de esta investigación es la de definir las competencias profesionales de tipo emocional y de personalidad de los maestros, a través la opinión de una muestra de profesionales en ejercicio. Posteriormente, evaluar las competencias que poseen una muestra de estudiantes de último curso de dicha titulación como futuros profesionales de magisterio, y comparar el perfil de competencias que poseen los alumnos con la opinión de los profesionales.

\section{MÉTODO}

\subsection{Participantes}

Para la presente investigación se dispuso de dos tipos de participantes: 
Una muestra de 148 maestros en ejercicio, que fueron seleccionados a través de un muestreo aleatorio estratificado de centros de educación infantil y primaria, entre el listado de centros disponible en Conselleria de Educación de la Generalitat Valenciana, perteneciente a la provincia de Alicante, y de titularidad tanto pública como privada/concertada. En el estudio participaron un total de 12 centros; 10 públicos y 2 concertados. La edad media de los maestros que han formado parte de este estudio es de 39 años, oscilando sus edades entre $22-66$. El 77\% pertenecen al género femenino y el $23 \%$ al masculino; siendo un total de 114 mujeres y 34 hombres.

Una muestra de estudiantes formada por alumnos de magisterio de último curso de carrera. Se incluyeron las especialidades de infantil, primaria, música y educación física impartidas en la Universidad de Alicante. El total de alumnos que formaron parte de este grupo fue de 139: 34 de magisterio infantil, 22 de primaria, 43 de magisterio musical y 40 de educación física. Las características demográficas de estos estudiantes son las siguientes: 32 alumnos de magisterio infantil, con edades comprendidas entre 20-25 años, con una edad media de 21 y el 100\% de ellos de género femenino; 20 alumnos de magisterio de primaria, con edades comprendidas entre 20-52 años, con una edad media de 25 y de género femenino el $86 \%$ y masculino el 14\%; 42 alumnos de magisterio musical, con edades comprendidas entre 20 32 años, con una edad media de 22 y de género femenino el $74 \%$ y masculino el 26\%; y 38 de magisterio de educación física, con edades comprendidas entre19-33 años, con una edad media de 23 y de género femenino el 55\% y masculino el $45 \%$.

\subsection{Instrumentos}

Instrumentos utilizados con los profesionales:

Para evaluar las competencias por los profesionales se empleó un cuestionario cuyo objetivo fue el de valorar 8 competencias relacionadas con la inteligencia emocional (atención emocional, claridad, reparación, inteligencia intrapersonal, inteligencia interpersonal, adaptación, control del estrés y humor) y 5 factores de personalidad (neuroticismo, extroversión, apertura, amabilidad y responsabilidad). Las competencias seleccionadas fueron extraídas a partir de tests administrados a los alumnos para evaluar la inteligencia emocional -TMMS-24 (Fernández-Berrocal, Extremera y Ramos, 2004) y EQ-i:S (Bar-On, 2002)- y la personalidad -NEO-FFI (Costa y McCrae, 1992).De este modo, se pudo comparar la opinión de los profesionales con las medidas tomadas a los estudiantes. 


\begin{tabular}{|c|c|}
\hline Competencia & Cuestión \\
\hline Atención & $\begin{array}{l}\text { ¿Qué atención deben prestar los maestros a su esta- } \\
\text { do anímico, problemas personales, inquietudes, etc.? }\end{array}$ \\
\hline Claridad & $\begin{array}{l}\text { ¿En qué medida puede influir en el trabajo cómo las } \\
\text { personas perciben y valoran sus emociones? }\end{array}$ \\
\hline Reparación & $\begin{array}{l}\text { ¿Qué creencia, opinión deben tener de su capacidad } \\
\text { para interrumpir y controlar los pensamientos ne- } \\
\text { gativos y aumentar los positivos? }\end{array}$ \\
\hline I. Intrapersonal & $\begin{array}{l}\text { ¿En que grado deben conocer sus emociones, ser ca- } \\
\text { paces de expresar lo que sienten y comunicar sus } \\
\text { necesidades a los otros? }\end{array}$ \\
\hline I. Interpersonal & $\begin{array}{l}\text { ¿Qué capacidad deben tener para establecer relacio- } \\
\text { nes cooperativas, constructivas y satisfactorias con } \\
\text { otras personas? (ser buenos oyentes; capaces de en- } \\
\text { tender y apreciar los sentimientos de los otros). }\end{array}$ \\
\hline Adaptación & $\begin{array}{l}\text { ¿A qué nivel necesitan manejar con éxito los cambios } \\
\text { y tener habilidades para solucionar los problemas } \\
\text { diarios enfrentándose a ellos de modo positivo? }\end{array}$ \\
\hline Manejo Estrés & $\begin{array}{l}\text { ¿En qué medida necesitan controlar sus impulsos y } \\
\text { trabajar bien bajo presión controlando el estrés? }\end{array}$ \\
\hline Humor & $\begin{array}{l}\text { ¿En qué grado necesitan tener un estado de ánimo } \\
\text { de felicidad y optimismo, ser energéticos y tener ca- } \\
\text { pacidad para auto-motivarse? }\end{array}$ \\
\hline Estabilidad Emocional* & $\begin{array}{l}\text { ¿En qué grado requieren estabilidad emocional, ca- } \\
\text { pacidad para mantener la calma y el control de sus } \\
\text { emociones en situaciones de estrés? }\end{array}$ \\
\hline Extroversión & $\begin{array}{l}\text { ¿En qué nivel deben ser energéticos y activos, poco } \\
\text { reservados y asertivos? (el extremo contrario sería } \\
\text { calmado, reservado, con tendencia al aislamiento) }\end{array}$ \\
\hline Apertura & $\begin{array}{l}\text { ¿Qué nivel de originalidad, imaginación, interés por } \\
\text { ideas nuevas y valores no convencionales requieren } \\
\text { para desarrollar con éxito su trabajo? }\end{array}$ \\
\hline Amabilidad & $\begin{array}{l}\text { ¿Qué grado de altruismo, generosidad, confianza y } \\
\text { solidaridad requieren para realizar su trabajo? (el } \\
\text { extremo contrario sería el escepticismo y el pensa- } \\
\text { miento crítico) }\end{array}$ \\
\hline Responsabilidad & $\begin{array}{l}\text { ¿En qué grado necesitan ser metódicos, ordenados y } \\
\text { escrupulosos en su labor profesional? }\end{array}$ \\
\hline
\end{tabular}

Tabla 1. Cuestionario sobre competencias socioemocionales para profesionales en ejercicio.

* La variable neuroticismo se ha cambiado al polo opuesto estabilidad emocional, en aras de la claridad para los profesionales. 
Instrumentos administrados a los estudiantes:

La Traid Meta-Mood Scale-24 (TMMS-24). Es una versión de la TMMS48 (desarrollado por Salovey y Mayer) adaptada y reducida por el grupo de investigación de Málaga (Fernández-Berrocal, Extremera y Ramos, 2004). Esta medida de auto-informe evalúa tres dimensiones clave de la inteligencia emocional: atención a los sentimientos, claridad emocional, y reparación de las emociones. A los sujetos se les pide que evalúen el grado en que están de acuerdo con cada uno de los ítems sobre una escala de tipo Likert de 5 puntos $(1=$ Nada de acuerdo, $5=$ Totalmente de acuerdo). Tras la reducción, la escala ha visto incrementada su fiabilidad en todos sus factores: Atención (.90); Claridad (.90) y Reparación (.86).

El Emotional Quotient Inventory: Short (EQ-i:S) de Reuven Bar-On (2002). Es una versión reducida del Emotional Quotient Inventory y adaptada al castellano por MHS, Toronto, Canadá. Consta de 51 ítems valorados en una escala tipo Likert de 5 puntos y evalúa cinco factores generales de la IE: inteligencia intrapersonal, inteligencia interpersonal, adaptación, gestión del estrés y humor general. El EQ-i:S muestra evidencias adecuadas de validez y la consistencia interna de sus subescalas oscila entre .65 y .86.

El Inventario de Personalidad NEO Reducido de Cinco Factores(NEOFFI) de Costa y McCrae (1992) -adaptado al castellano por TEA ediciones en 2002-. Es un instrumento que evalúa los cinco grandes factores de personalidad y que ofrece de forma abreviada una medida de las dimensiones: Neuroticismo, Extraversión, Apertura, Amabilidad y Responsabilidad. Consta de 60 ítems y su rango de opciones de respuesta va desde A (Totalmente en desacuerdo) a E (Totalmente de acuerdo). El desarrollo y validación del cuestionario muestra fiabilidad de consistencia interna con valores que oscilan entre .86 y .95 y estabilidad test-retest con valores que oscilan entre .70 y .92 -en la muestra española-, así como validez factorial.

\subsection{Procedimiento}

Para recopilar la información procedente de los titulados incorporados al mundo laboral se hizo, en primer lugar, un sorteo de 20 centros entre la relación de centros de la provincia de Alicante disponible en la página web de Conselleria de Educación. Estos centros fueron ordenados según el orden del sorteo y se seleccionaron los 10 primeros para comenzar el estudio. Una vez realizada la selección de centros participantes en el estudio, se procedió a la difusión de los cuestionarios entre el profesorado. Para ello se contactó con los directores de los centros o en su defecto con los jefes de estudio, quienes se encargaron de distribuir el cuestionario entre todos los ma- 
estros y se dejó un plazo aproximado de una semana para su ejecución. Posteriormente, para recopilar los cuestionarios elaborados, se utilizaron los dos métodos a elección de los equipos directivos: en unos casos se proporcionó sobre y sello con la dirección del destinatario para su envío por correo y, en otros, se retornó a los centros para su recogida. Dependiendo de los cuestionarios recopilados se fue ampliando la muestra añadiendo centros en el mismo orden del sorteo, hasta conseguir una muestra de similar tamaño al grupo de estudiantes de magisterio con el que posteriormente se compararían los resultados.

Para recopilar la información procedente de los estudiantes de magisterio se contactó directamente con parte del profesorado de las asignaturas de último curso de carrera, de cada especialidad. Las asignaturas escogidas fueron las que ofrecían un alto número de alumnos siendo de naturaleza troncal. Una vez dado el consentimiento por el profesorado, se eligieron las fechas oportunas para poder realizar los tests en hora de clase, a fin de que el número de alumnos por aula fuera el máximo posible. La recolección de datos se realizó durante el primer trimestre del curso, tanto en horario de mañana como de tarde, aplicando las pruebas en sus respectivas aulas. Para realizar los tests se les concedió a los sujetos el tiempo necesario; otorgándoles un tiempo máximo para todas las pruebas de dos horas aproximadamente.

\subsection{Diseño y análisis de datos}

En la presente investigación se han empleado diferentes técnicas de análisis de datos, dentro de un diseño que puede considerarse,de forma general, ex post facto de tipo comparativo.

En primer lugar se establecieron los perfiles de cada grupo (profesionales y estudiantes) tomando en consideración las distintas variables medidas. En el caso de los profesionales, el perfil se estableció a través de la media de las puntuaciones otorgadas en los cuestionarios, para las distintas variables. Para obtener el perfil en los alumnos, se les evaluó a través de la aplicación de diferentes pruebas y se convirtieron las puntuaciones de todas las variables a una escala de 1 a 10 puntos lo que posibilitó la comparación directa con las puntuaciones dadas en el cuestionario aplicado a profesionales. Por último se estableció la media de cada variable evaluada.

Para realizar el análisis estadístico de la comparación de los perfiles de ambos grupos (maestros y estudiantes de magisterio) se ha utilizado el módulo MLG (Modelo Lineal General) del paquete estadístico SPSS versión 15.0. Con este procedimiento se realiza un análisis de varianza multivariado 
(MANOVA) y un análisis de varianza -ANOVA- univariado, de medidas repetidas, en el que las medidas de las variables socioemocionales se tratan como variables medidas dentro de los mismos sujetos, y los grupos (profesionales y estudiantes de magisterio) actúan como variables entre sujetos; procedimiento comúnmente empleado en el análisis de perfiles (Tabachnick \& Fidell, 2007).

\section{RESULTADOS}

La Tabla 2 muestra las medias, desviaciones típicas y número de casos, tanto para el grupo de estudiantes como de profesionales. Las puntuaciones medias de los estudiantes oscilan entre 6,81 y 8,85 para las variables de atención e inteligencia interpersonal respectivamente.

Así mismo, si la media más alta corresponde a la variable inteligencia interpersonal, es esta misma variable la que posee la desviación típica más

\begin{tabular}{|l|c|c|c|c|c|c|c|c|c|}
\hline & \multicolumn{3}{|c|}{ Alumno } & \multicolumn{2}{c|}{ Profesional } & \multicolumn{3}{c|}{ Total } \\
\hline & & $\mathrm{s}$ & $\mathrm{N}$ & & $\mathrm{s}$ & $\mathrm{N}$ & & $\mathrm{s}$ & $\mathrm{N}$ \\
\hline Atención & 6,81 & 1,43 & 132 & 6,79 & 2,68 & 137 & 6,80 & 2,15 & 269 \\
\hline Claridad & 6,86 & 1,44 & 132 & 7,78 & 1,86 & 137 & 7,33 & 1,72 & 269 \\
\hline Reparación & 6,90 & 1,43 & 132 & 8,28 & 1,31 & 137 & 7,60 & 1,53 & 269 \\
\hline Intrapersonal & 7,53 & 1,15 & 132 & 8,42 & 1,28 & 137 & 7,98 & 1,29 & 269 \\
\hline Interpersonal & 8,85 &, 74 & 132 & 9,29 &, 85 & 137 & 9,07 &, 83 & 269 \\
\hline Adaptación & 7,59 & 1,07 & 132 & 8,85 &, 89 & 137 & 8,23 & 1,17 & 269 \\
\hline M Estrés & 7,42 & 1,23 & 132 & 8,86 &, 92 & 137 & 8,15 & 1,30 & 269 \\
\hline Estado Ánimo & 7,90 & 1,09 & 132 & 8,90 & 1,01 & 137 & 8,41 & 1,16 & 269 \\
\hline Estabilidad Emocional* & 5,62 & 1,32 & 132 & 8,91 &, 91 & 137 & 7,29 & 1,99 & 269 \\
\hline Extroversión & 7,75 & 1,07 & 132 & 7,94 & 1,29 & 137 & 7,85 & 1,19 & 269 \\
\hline Apertura & 6,99 & 1,00 & 132 & 8,50 & 1,08 & 137 & 7,76 & 1,28 & 269 \\
\hline Amabilidad & 7,54 &, 84 & 132 & 8,51 & 1,13 & 137 & 8,03 & 1,10 & 269 \\
\hline Responsabilidad & 7,53 &, 98 & 132 & 8,36 & 1,19 & 137 & 7,95 & 1,17 & 269 \\
\hline
\end{tabular}

Tabla 2. Estadísticos descriptivos para cada grupo y el total

*La puntuación de los estudiantes en la variable neuroticismo se ha invertido a estabilidad emocional, porque es requisito del procedimiento estadístico utilizado para el análisis de perfiles que todas las variables sigan la misma tendencia. 
baja $(s=, 74)$ y la más alta, la claridad $(s=1,44)$, que está muy próxima a la variable atención a las propias emociones $(\mathrm{s}=1,43)$.

En cuanto a las puntuaciones medias de los profesionales, éstas fluctúan entre 6,79 y 9,29 coincidiendo las medias más altas en las mismas variables que los alumnos: atención e inteligencia interpersonal respectivamente. También es la variable inteligencia interpersonal la que posee la desviación típica más baja $(\mathrm{s}=, 85)$ y la atención la que tiene la más alta $(s=2,68)$, seguida a una gran distancia por el resto de variables $(s=1,86$ es la posterior inmediata).

Para la mayoría de las variables existe una distancia de aproximadamente 1 punto entre la media de los alumnos y la de los profesionales, correspondiendo la mayor puntuación a estos últimos. Esto no se cumple en la variable estabilidad emocional cuya distancia supera los 3 puntos y la atención que es mayor en los alumnos y la distancia entre ellas es mínima (,02).

El resultado de la prueba $\mathrm{M}$ de Box no muestra homogeneidad de las matrices de varianza covarianza $\left(\mathrm{F}_{(91,222797)}=3,502 \mathrm{y} \mathrm{p}=, 000\right)$; sin embargo la violación de este supuesto tiene un mínimo impacto si los grupos son aproximadamente de igual tamaño (Hair, et al, 1999) o la mayor ratio de la varianza entre los grupos $(1: 3,48)$ no supera la ratio 1:10 (considerado el límite máximo en el análisis de perfiles para Tabachnick y Fidell, 2007).

La prueba de Mauchly arroja un valor bajo, $\mathrm{W}=, 200$, lo que se corresponde con un $\chi^{2}=1022,28$ para $\mathrm{gl}=77 \mathrm{y} \mathrm{p}=, 000$ no cumpliéndose por tanto el supuesto de esfericidad de la matriz de las VDs. Por consiguiente, el test intra-sujetos para los efectos de planitud (efecto socioemocional) y paralelismo (efecto de interacción socioemocional*grupo) se ha realizado con los grados de libertad corregidos con los valores de ajuste de Epsilon. Aunque los valores de Epsilon, calculados según los procedimientos de Greenhouse-Geisser $\varepsilon=, 083$,Huynh-Feldt $\varepsilon=0,498$, y Límite inferior $\varepsilon=0,513$, fueron bajos, una vez hechas estas correcciones, como se puede observar en la tabla 3 , en todos los casos las razones Fs resultan altamente significativas, $\mathrm{p}<.001$, tanto para el efecto intrasujetos, como para el efecto de interacción.

En esta misma tabla se puede observar el resultado del análisis de los efectos intra-sujetos resultando perfiles no planos y no paralelos. Las variaciones de las VDs han resultado significativas, para todos los estadísticos, en el test de planitud $(F=68,80 ; p<, 001$ y h2 parcial $=, 20)$. Estos perfiles se muestran de forma gráfica en la Figura 1. También han resultado significativas las variaciones de las variables socioemocionales para cada grupo en todos los estadísticos correspondientes al test de paralelismo $(\mathrm{F}=34,88 ; \mathrm{p}$ $<, 001$ y h2 parcial =,11). 


\begin{tabular}{|c|c|c|c|c|c|c|c|c|}
\hline Fuente & & $\begin{array}{l}\text { Suma de } \\
\text { cuadra- } \\
\text { dos tipo } \\
\text { III }\end{array}$ & Gl & $\begin{array}{c}\text { Media } \\
\text { cuadráti- } \\
\text { ca }\end{array}$ & $\mathbf{F}$ & Sig. & $\begin{array}{c}\text { h2par- } \\
\text { cial }\end{array}$ & $\begin{array}{l}\text { Poten- } \\
\text { cia ob- } \\
\text { serva- } \\
\text { da (a) }\end{array}$ \\
\hline \multirow[t]{4}{*}{$\begin{array}{c}\text { socioemocio- } \\
\text { nal }\end{array}$} & $\begin{array}{l}\text { Esfericidad } \\
\text { asumida }\end{array}$ & 1039,10 & 12 & 86,59 & 68,80 & ,000 & ,20 & 1,00 \\
\hline & $\begin{array}{l}\text { Greenhouse- } \\
\text { Geisser }\end{array}$ & 1039,10 & 5,98 & 173,71 & 68,80 & ,000 & ,20 & 1,00 \\
\hline & Huynh-Feldt & 1039,10 & 6,15 & 168,77 & 68,80 & 000 & 20 & 1,00 \\
\hline & Límite-inferior & 1039,10 & 1,00 & 1039,10 & 68,80 & ,000 & ,20 & 1,00 \\
\hline \multirow{4}{*}{$\begin{array}{l}\text { socioemocio- } \\
\text { nal } \\
* \text { grupo }\end{array}$} & $\begin{array}{l}\text { Esfericidad } \\
\text { asumida }\end{array}$ & 526,80 & 12 & 43,90 & 34,88 & ,000 & ,11 & 1,00 \\
\hline & $\begin{array}{l}\text { Greenhouse- } \\
\text { Geisser }\end{array}$ & 526,80 & 5,98 & 88,07 & 34,88 & ,000 & ,11 & 1,00 \\
\hline & Huynh-Feldt & 526,80 & 6,15 & 85,56 & 34,88 & ,000 & ,11 & 1,00 \\
\hline & Límite-inferior & 526,80 & 1,00 & 526,80 & 34,88 & ,000 & ,11 & 1,00 \\
\hline \multirow{4}{*}{$\begin{array}{c}\text { Error } \\
\text { (socioemocio- } \\
\text { nal) }\end{array}$} & $\begin{array}{l}\text { Esfericidad } \\
\text { asumida }\end{array}$ & 4032,07 & 3204 & 1,25 & & & & \\
\hline & $\begin{array}{l}\text { Greenhouse- } \\
\text { Geisser }\end{array}$ & 4032,07 & 1597,07 & 2,52 & & & & \\
\hline & Huynh-Feldt & 4032,07 & 1643,86 & 2,45 & & & & \\
\hline & Límite-inferior & 4032,07 & 267,00 & 15,10 & & & & \\
\hline
\end{tabular}

Tabla 3. Pruebas de efectos intra-sujetos.

a Calculado con alfa $=, 05$ 


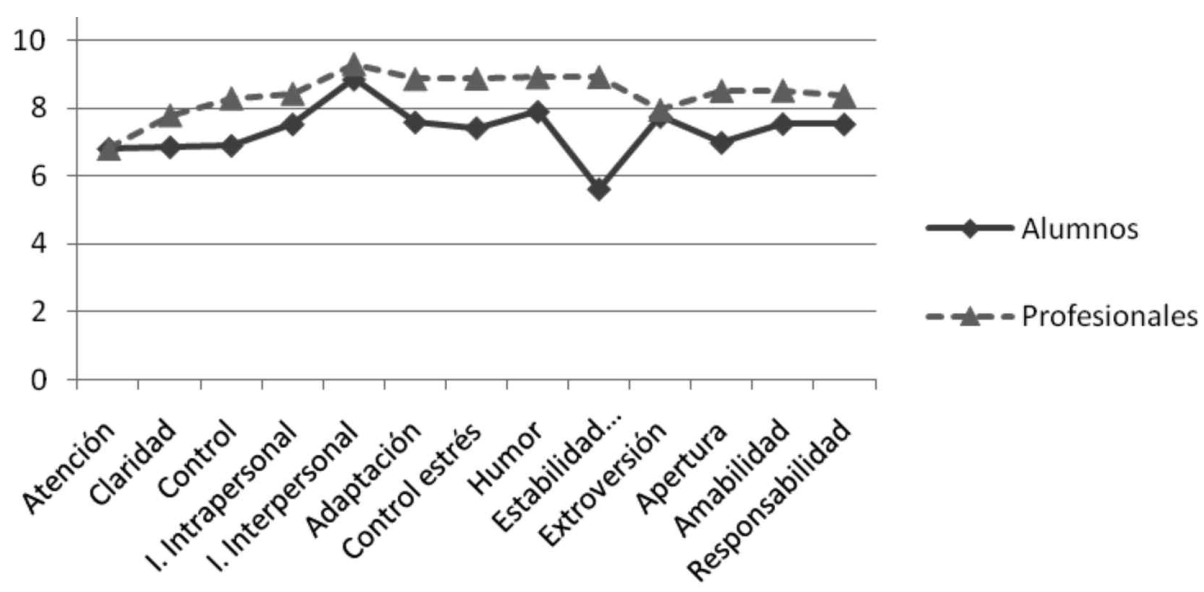

Figura 1. Gráfica de los perfiles de profesionales y estudiantes de magisterio.

Para evaluar las diferencias entre los grupos, en cada una de las variables, se realiza una prueba $t$ de diferencia de medias para grupos independientes. La Tabla 4 proporciona los resultados de esta prueba.

\begin{tabular}{|l|c|c|c|c|}
\hline \multicolumn{1}{|c|}{ Variable } & $\begin{array}{c}\text { Media de los } \\
\text { alumnos }\end{array}$ & $\begin{array}{c}\text { Media de los } \\
\text { profesionales }\end{array}$ & t & p \\
\hline Atención & 6,81 & 6,79 & $-0,234(1)$ & 0,815 \\
\hline Claridad & 6,86 & 7,78 & $-4,956$ & 0,000 \\
\hline Reparación-Control & 6,90 & 8,28 & $-8,455$ & 0,000 \\
\hline Intrapersonal & 7,53 & 8,42 & $-6,379$ & 0,000 \\
\hline Interpersonal & 8,85 & 9,29 & $-4,249$ & 0,000 \\
\hline Adaptación & 7,59 & 8,85 & $-10,493$ & 0,000 \\
\hline M Estrés & 7,42 & 8,86 & $-10,763(1)$ & 0,000 \\
\hline Estado Ánimo & 7,90 & 8,90 & $-8,243$ & 0,000 \\
\hline Estabilidad Emoc. & 5,62 & 8,91 & $-23,524(1)$ & 0,000 \\
\hline Extroversión & 7,75 & 7,94 & $-1,627$ & 0,105 \\
\hline Apertura & 6,99 & 8,50 & $-12,463$ & 0,000 \\
\hline Amabilidad & 7,54 & 8,51 & $-8,189(1)$ & 0,000 \\
\hline Responsabilidad & 7,53 & 8,36 & $-6,337$ & 0,000 \\
\hline
\end{tabular}

Tabla 4. Resultados de la prueba t de diferencia de medias entre ambos grupos en cada una de las variables.

(1) No se asumen varianzas iguales a partir de la prueba de Levene. 
Como se puede observar, sólo en las variables Atención y Extroversión no aparecen diferencias significativas entre los grupos; mientras que en las variables Claridad, Reparación, Inteligencia intrapersonal, Inteligencia interpersonal, Adaptación, Manejo del Estrés, Estado de Ánimo, Estabilidad Emocional, Apertura, Amabilidad y Responsabilidad aparecen diferencias estadísticamente significativas entre los grupos; en todas ellas los profesionales muestran una media mayor que los estudiantes.

En cuanto al test de nivel (Tabla 5), se puede comprobar que existen diferencias entre las medias de los grupos en las variables socioemocionales. El coeficiente del factor de pertenencia al grupo (tamaño del efecto $\mathrm{h}^{2}$ parcial $=, 40$ ) explica la gran diferenciación de los parámetros socioemocionales entre los grupos.

\begin{tabular}{|c|c|c|c|c|c|c|c|}
\hline Fuente & $\begin{array}{c}\text { Suma de } \\
\text { cuadrados } \\
\text { tipo III }\end{array}$ & gl & $\begin{array}{c}\text { Media } \\
\text { cuadrática }\end{array}$ & F & Sig. & $\begin{array}{c}\text { h2 } \\
\text { parcial }\end{array}$ & $\begin{array}{c}\text { Potencia } \\
\text { observada } \\
\text { (a) }\end{array}$ \\
\hline Intersección & 216892,73 & 1 & 216892,73 & 38310,95 &, 000 &, 99 & 1,00 \\
\hline grupo & 1030,38 & 1 & 1030,38 & 182,00 &, 000 &, 40 & 1,00 \\
\hline Error & 1511,58 & 267 & 5,66 & & & & \\
\hline
\end{tabular}

Tabla 5. Pruebas de los efectos inter-sujetos a Calculado con alfa $=, 05$

\section{DISCUSIÓN Y CONCLUSIONES}

El estudio revela que existen diferencias entre las competencias socioemocionales de los estudiantes y aquellas que los profesionales opinan que deben poseer para desarrollar su labor exitosamente. De hecho, las puntuaciones medias de los profesionales están casi siempre por encima de las competencias que poseen los estudiantes. Además, el elevado tamaño del efecto, eta al cuadrado, pone de manifiesto de forma clara la existencia de estas diferencias significativas entre las medias de los estudiantes y los maestros integrados en el mercado laboral. 
Los resultados muestran que los estudiantes no están preparados para una integración laboral exitosa en cuanto a competencias socioemocionales se refiere: no tienen las habilidades suficientes para trabajar en equipo, en el manejo de personas, en adaptarse a los continuos cambios, o en controlar sus emociones entre otras. Estos resultados están en consonancia con los resultados obtenidos en el Proyecto Reflex (ANECA, 2007) para las competencias profesionales, en el cual se destaca que los empleadores demandan más competencias de las adquiridas por los graduados, mientras éstos dicen usar poco las competencias poseídas; destacando la diferencia en el nivel de competencias necesarias para el desempeño profesional y en el nivel de competencias adquiridas en la educación, así como la poca utilización que, de las competencias adquiridas, hacen uso los titulados en el puesto de trabajo.

También, tomando en consideración la media de las puntuaciones otorgadas por los profesionales a todas las variables podemos comprobar que todas las competencias socioemocionales estudiadas en esta investigación son relevantes, en mayor o menor medida, para el desarrollo profesional de los maestros puesto que estas medias siempre están por encima de 5 .

Como se ha podido comprobar por la opinión de los profesionales, los estudiantes necesitan desarrollar más competencias genéricas entre las que se hallarían las socioemocionales. Si las universidades pretenden formar a sus alumnos en las competencias demandadas por las empresas, la formación inicial del docente deberá incluir las competencias emocionales si queremos ser coherentes con lo que la investigación nos ha enseñado, con lo que las leyes educativas nos exigen y con el modelo de sociedad europea que perseguimos (Palomera, Fernández-Berrocal y Brackett, 2008).

Por otro lado, recientes investigaciones afirman que los directivos que demuestran ser "Altamente Efectivos", comienzan a desarrollar sus competencias a edades tempranas y antes de su experiencia laboral (Dreyfus, 2008). Sin embargo, aunque el consenso sobre la importancia de las competencias emocionales es alto (Palomera, Fernández-Berrocal y Brackett, 2008), desarrollar esta formación depende de toda la comunidad educativa que interviene en el proceso, desde la escuela a la universidad, y tanto una como otra se encuentra a menudo con grandes dificultades (Elias et al., 1997; Zins, Weissberg, Wang \&Walberg, 2004). Además, como señala Boyatzis (2008), existen pocos estudios sobre el desarrollo de competencias.

Castejón, Cantero y Pérez (2008) aprecian la importancia que tiene la identificación, evaluación y el desarrollo de las competencias emocionales en el contexto educativo de la enseñanza superior universitaria partiendo de la consideración de que, por un lado, las habilidades comprometidas en la in- 
teligencia emocional, en cuanto que están relacionadas con el desempeño en el ámbito personal, profesional y en la vida diaria en general, constituyen modelos de competencias (Boyatzis, 1999; Boyatzis, Goleman y Rhee, 2000; Hendlund y Sternberg, 2000); y por otro lado, los modelos de inteligencia emocional incluyen competencias amplias de carácter socio-emocional (Mayer, Salovey y Caruso, 2000).

La cuestión que se plantea es la forma de incorporar las competencias genéricas de tipo sociomocional, dentro de los currícula universitarios establecidos en el marco del Espacio Europeo de Educación Superior. Aunque existen varias iniciativas en este sentido (Fallows y Stevens, 2000; Jaeger, 2003), éstas se han puesto en marcha en ámbitos distintos al educativo.

\section{NOTAS}

Esta investigación ha sido financiada por el Proyecto de I+D PSI200912696 de la Secretaría de Estado de Investigación, con el título: Competencias Intelectuales, Personales y Socioemocionales en la Inserción Laboral de los Egresados Universitarios. 


\section{REFERENCIAS BIBLIOGRÁFICAS}

Agencia Nacional de Evaluación de la Calidad y Acreditación (ANECA) (2005). Libro Blanco del Título de Grado en Magisterio. Volumen 1. Disponible en: http://www.aneca.es/media/150404/libroblanco jun05 magiste rio1.pdf

Agencia Nacional de Evaluación de la Calidad y Acreditación (ANECA). (2007). Proyecto Reflex. Informe Ejecutivo.

Aiken, L.H.; Clarke, S.P.; Sloane, D.M., y Sochalski, J. (2002), Hospital Nurse Staffing and Patient Mortality, Nurse Burnout, and Job Dissatisfaction. JAMA, 2002 - American Medical Association, 288(16), 1987-1993.

Aluja, A.; Blanch, A. y Biscarri, J. (2002). Síndrome de Quemarse en el trabajo (Burnout) y Estrategias de Afrontamiento: un modelo de relaciones estructurales. Revista de Psicología del Trabajo y de las Organizaciones, 18(1), 57-74.

Bar-On, R. (2000). Emotional and social intelligence: insights from emotional quotient inventory, en Bar-On, R. y Parker J. D.A. (Eds.) The handbook of emotional intelligence: Theory, development, assessment, and application at home, school, and in the workplace. San Francisco: Jossey-Bass, 343-362.

Bar-On, R. (2002). EQ-i:S Bar On Emocional Quotient Inventory: Technical Manual. Toronto: Multi-HealthSystems.

Bisquerra, R. (2005). La educación emocional en la formación del profesorado. Revista Interuniversitaria de Formación del Profesorado, 19 (3), 95-114.

Bisquerra, R. y Pérez, N. (2007). Las competencias emocionales. Monográ- fico: Orientación y formación en competencias. Educación XX1, 10.

Boyatzis, R.E. (1999). Self-directed change and learning as a necessary metacompetency for success and effectiveness in the 21st century, en Sims, R. \&. Veres, J.G. (Eds.) Keys to employee success in the coming decades. Westport: Greenwood,15-32.

Boyatzis, R.E. (2008). Competencies in the 21 st century. Journal of Management Development, 27 (1), 5-12.

Boyatzis, R.E., Goleman D. y Rhee, K. (2000). Clustering competente in emotional intelligence: insights from the emotional competence inventory (ECI), en Bar-On, R. \& Parker, J. D. A. (Eds.) Handbook of Emotional Intelligence. San Francisco: Jossey-Bass, 343362.

Brotheridge, C.M. y Lee, R.T. (2008). The emotions of managing: An introduction to the special issue. Journal of Managerial Psychology, 23 (2), 108-117.

Bueno, C., Teruel, M.P., y Valero, A. (2005). La inteligencia emocional en alumnos de Magisterio: La percepción y comprensión de los sentimientos y las emociones. Revista Interuniversitaria de Formación del Profesorado, 19 (3), 169-194.

Byron, C.M. (2001). The effects of emotional knowledge education in the training of novice teachers. Dissertation Abstract: 2001-95021-143.

Cano, E. (2005). Commillorar les competències dels docents. Barcelona: Graó.

Castejón, J. L., Cantero, M ${ }^{\mathrm{a}}$ P. y Pérez, N. (2008). Diferencias en el perfil de competencias socio-emocionales en estudiantes universitarios de diferentes ámbitos científicos. Revista electróni- 
ca de Investigación Psicoeducativa,6(2), 339-362.

Chan, D. W. (2006). Emotional Intelligence and components and burnout among Chinese secondary school teachers in Hong Kong. Teaching and teacher education, 22 (8), 1042- 1054.

Chan, D.W. (2008). Emotional intelligence, self-efficacy, and coping among Chinese prospective and in-service teachers in Hong Kong. Educational Psychology, 28 (4), 397-408.

Cherniss, C. (2000). Emotional Intelligence: What it is and Why it Matters. Rugters University. Ensayo presentado en el Annual Meeting of the Society for Industrial and Organizational Psychology, Nueva Orleans.

Ciarrochi, J. V., Chan, A.C., y Caputi, P. (2000). A critical evaluation of the emotional intelligence construct. Personality and Individual Difference, 28 (3), 539-561.

Ciarrochi, J.V., Deane, F., y Anderson, S. (2002). Emotional intelligence moderates the relationship between stress and mental health. Personality and Individual Differences, 32, 197-209.

Cooper, R.K. (1997). Applying emotional intelligence in the workplace. Training \& Development, 51 (12), 31-39.

Costa, P, y McCrae, R. (2002). Inventario de Personalidad Reducido de Cinco Factores(NEO-FFI). Madrid: TEA Ediciones.

Culver, R. y Yokomoto, Ch. (1999). Optimum Academic Performance and its Relation to Emotional Intelligence. 29th ASEE/IEEE Frontiers in Education Conference, San Juan, Puerto Rico.

Di Fabio, A. y Palazzeschi, L. (2008). Emotional intelligence and self-effi- cacy in a sample of Italian high school teachers. Social Behavior and Personality, 36(3), 315-325.

Dreyfus, C.R., (2008). Identifying competencies that predict effectiveness of R\&D managers. Journal of Management Development, 27(1), 76-91.

Durán, M.A., Montalbán, F.M., Rey, L, Extremera, N. (2005). Engagement y burnout en el ámbito docente: análisis de sus relaciones con la satisfacción laboral y vital en una muestra de profesores. Revista de Psicología del Trabajo y de las Organizaciones, 21 (12), 145-158.

Elias, M., Zins, J., Weissberg, R., Frey, K., Greenberg, T., Haynes, N., Kessler, R., Schwab-Stone, M., y Shriver, T. (1997). Promoting social and emotional learning: Guidelines for educators. Alexandria, VA: Association for Supervision and Curriculum Development.

Extremara, N., y Fernández-Berrocal, P. (2004). La importancia de desarrollar la inteligencia emocional en el profesorado. Revista Iberoamericana de Educación, 33(8). Disponible en: http:// www.campus-oei.org/revista/deloslectores/759Extremera.PDF [Consulta 2010, de 20 enero].

Extremera, N., Fernández-Berrocal, P., Mestre, J. y Guil, R. (2004). Medidas de evaluación de la Inteligencia Emocional. Revista Latinoamericana de Psicología, 36 (2), 209-228.

Fallows, S., y Stevens, C. (2000). Integrating key skills in higher education: employability, transferable skills and learning for life. Londres: Kogan Page.

Fernández-Berrocal, P., Extremera, N. y Ramos, N. (2004). Validity and reliability of the spanish modified version of The Traid Meta-Mood Sacale. Psychological Reports, 94, 751-755. 
Gil Flores, J. (2007). La Evaluación De Competencias Laborales. Monográfico: Orientación y Formación en Competencias. Educación XX1, 10.

Gil-Monte, P. R. (1991). Burnout en enfermería: un estudio comparativo España-Holanda, Revista de Psicología del Trabajo y de las Organizaciones, 7(19), 121-130.

Goleman, D. (1995). Emotional Intelligence. Nueva York: Bantam Books.

Goleman, D. (1998). Working with Emotional Intelligence. Nueva York: Bantam Books.

Goleman, D. (2001). An EI-based theory of performance, en Cherniss, C. \&Goleman, D. (Eds.) The Emotionally Intelligent Workplace. San Francisco: Jossey-Bass,27-44.

González, J. y Wagenaar, R. (Eds.) (2003). Tuning Educational Structures in Europe. Informe final. Fase Uno. Bilbao: Universidad de Deusto y Universidad de Groningen.

Hair, J. F., Anderson, RE., Tatham, R. L., y Black, WC. (1999). Análisis Multivariante ( $5^{\mathrm{a}}$ Ed.) Madrid: Prentice Hall Iberia.

Hendlund, J. y Sternberg, R. J. (2000). Too many intelligences? Integrating social, emotional and practical intelligence, en Bar-On, R.y. Parker, J. D. A (Eds.) The handbook of emotional intelligence. San Francisco: Jorsey-Bass, 136-168.

Jaeger, A. (2003). Job competencies and the curriculum: An inquiry into emotional intelligence in graduate professional education. Research in Higher Education, 44 (6), 615-639.

Jennings, P. A. y Greenberg, M. T. (2009). The Prosocial Classroom: Teacher Social and Emotional Competence in Re- lation to Student and Classroom Outcomes. Review of Educational Research, 79 (1), 491-525.

Koman, E. S. y Wolff, S. B. (2008). Emotional intelligence competencies in the team and team leader: A multi-level examination of the impact of emotional intelligence on team performance. Journal of Management Development, 27 (1), 55-75.

Lam, L. T. (1998). Emotional Intelligence: Implications for Individual Performance. (Abstract) Texas TechUniversity. Recuperado de la Base de Datos ProQuest, CD-ROM

Landa, J. M. A., Lopez-Zafra, E., de Antonana, R., Pulido, M. (2006). Perceived emotional intelligence and life satisfaction among university teachers. Psicothema, 18, 152-157.

Matthews, G., Zeidner, M., y Robert, R. D. (2002). Emotional intelligence: Science and myth. Cambridge, MA: MIT Press.

Mayer, J. D., Caruso, D., y Salovey, P. (2000). Selecting a measure of emotional intelligence, en Bar-On, R. y Parker, J. D. A, (Coords.) Handbook of emotional intelligence. Theory, development, assessment, and application at home, school and in the workplace. San Francisco: Jossey-Bass, 301-319.

Mayer, J. D. y Salovey, P. (1997). What is emotional intelligence?, en Salovey, P. y Sluyter, D. (Eds.) Emotional development and emotional intelligence: educational applications. Nueva York: Basic Books, 3-31.

Mayer, J. D., Caruso, D. R., y Salovey, P. (1999). Emotional intelligence meets traditional standards for an intelligence. Intelligence, 27 (4), 267-298.

Mayer, J. D., Caruso, D. R. y Salovey, P. (2002). Mayer-Salovey-Caruso Emotio- 
nal Intelligence Test (MSCEIT): User's Manual. Toronto, Canada: MHS Publisher.

Mayer, J. D., Salovey, P. y Caruso, D. (2000). Models of emotional intelligence, en Sternberg, R.J. (Ed.) Handbook of Intelligence. $2^{\text {a }}$ Ed. Nueva York: Cambridge University Press, 396-420.

McClelland, D. C. (1973). Testing for competence rather than intelligence, American Psychologist, 28 (1), 1-14.

Mestre, J. M., Guil, M.R. y Gil-Olarte, P. (2004). Inteligencia Emocional: algunas respuestas empíricas y su papel en la adaptación escolar en una muestra de alumnos de secundaria. Revista Electrónica de Motivación y Emoción (REME), VI (16).

Møller, C. y Powell, S. (2001). Emotional Intelligence and the challenges of quality management today. Leader ship \& Organization Development Journal, 27 (7), 341-344.

Murga, J. y Ortego, E. (2003). La importancia de la inteligencia emocional en el funcionamiento de las organizaciones. Encuentros en Psicología Social, 1 (4), 79-82.

Organización Internacional del Trabajo: OIT/CINTERFOR. Banco de herramientas para competencia laboral. Disponible en:

http://www.ilo.org/public/spanish/region/ampro/cinterfor/temas/complab/banco/index.htm [consulta: 2008, 1 de Abril de 2008]

Organización para la Cooperación y el Desarrollo Económico (OCDE) (2002). Definition and selection of Competences (DeSeCo): Theoretical and Conceptual Foundations. Estrategy Paper of Directorate for Education, Employment, Labour and Social Affairs. Education Committee.
Palomera, R., Fernández-Berrocal, P. y Brackett, M.A. (2008). La inteligencia emocional como una competencia básica en la formación inicial de los docentes: algunas evidencias. Revista Electrónica de Investigación Psicoeducativa, 15(6-2), 437-454.

Parker, J. D. A. (2002). Emotional Intelligence and Academic Success: Examining the Transition from High School to University. Annual meeting of the $\mathrm{Ca}$ nadian Psychological Association, Vancouver: British Columbia.

Parker, J. D. A., Taylor, G. J. y Bagby, R. M. (2001). The relationship between emotional intelligence and alexithymia. Personality and Individual Differences, 30 (1), 107-115.

Pesquero, E., Sánchez, M.E., González, M., y Martín, R. (2008). Las competencias profesionales de los maestros de Primaria. Revista Española de Pedagogía, 241, 447-466.

Petrides, K.V., y Furham, A. (2003). Trait emotional intelligence: Behavioral validation in two studies of emotion recognition and reactivity to mood induction. European Journal of Personality, 17, 39-57.

Repetto E., y Pérez-González, J.C. (2007). Formación en competencias socioemocionales a través de las prácticas en empresas. Revista Europea de Formación Profesional, 40 (1), número Monográfico.

Rozeil, E. J., Pettijohn, Ch. E. y Parker, R. S. (2001). An empirical evaluation of emotional intelligence. The impact on management development. Journal of Managerial Development, 21(4), 272289.

Sala, J., y Abarca, M. (2002). Las competencias emocionales de los futuros profesores/as. Revista Electrónica Interu- 
niversitaria de Formación del Profesorado, 5 (3).

Salovey P. y Mayer J. (1990). Emotional Intelligence. Imagination, Cognition, and Personality, 9, 185-211.

Salovey, P. (2001). Applied emotional intelligence: Regulating emotions to become healthy, wealthy, and wise, en Ciarrochi, J.;Forgas, J. P. y Mayer, J.D. (Eds.) Emotional Intelligence in Everyday Life: A Scientific Inquiry. Filadelfia: Psychology Press/Taylor y Francis Group, 168-184.

Schutte, N. S., Malouff, J., Hall, L. E., Haggerty, D. J., Cooper, J.T., Golden, C. J. y Dornheim, L. (1998). Development and validation of a measure of emotional intelligence. Personality and Individual Differences, 25, 167-177.

Sjöberg, L. (2001). Emotional intelligence: A psychometric analysis. European Psychologist, 6 (2), 79-95.

Sutton, R. E. y Wheatley, K. F. (2003). Teacher's Emotions and Teaching: A review of the literature and directions for future research. Educational Psychology Review, 15 (4), 327-358.

Tabachnick, B. y Fidell, L. (2007). Using multivariate statistics. $5^{\text {a }}$ Ed.: Boston: Allyn \& Bacon.

Teruel, M.P. (2000). La inteligencia emocional en el currículo de la formación inicial de los maestros. Revista Interuniversitaria de Formación del Profesorado, 38, 141-152.
UNESCO (1996). La educación encierra un tesoro. Informe a la UNESCO de la Comisión Internacional sobre la Educación para el siglo XXI. (Informe Delors). Madrid: Santillana/Ediciones UNESCO.

Vanderberghe, J. y Huberman, A. M. (Eds.) (1999). Understanding and preventing teacher burnout: A sourcebook of international research and practice. Cambridge, UK: Cambridge University Press.

Vivas de Chacón, M. (2004). Las competencias socio-emocionales del docente: una mirada desde los formadores de formadores. I Jornadas Universitarias sobre Competencias Socio-Profesionales de las Titulaciones de Educación, Madrid: UNED.

Weare, K. y Gray, G. (2003). What works in developing children's emotional and social competence and wellbeing? Department for Education and Skills Research Report, 456. Londres: DfES.

Yoon, J. S. (2002). Teacher characteristics as predictors of teacher-student relationships: stress, negative affect, and self-efficacy. Social Behavior and Personality: An International Journal, 30 (5), 485-493.

Zins, J. E., Weissberg, R. P., Wang, M. C., y Walberg, H. J. (Eds.) (2004). Building academic success on social and emotional learning. Nueva York: Teachers College Press 


\title{
PALABRAS CLAVE
}

Competencias; Competencias socio-emocionales; Educación del profesor basada en competencias; Inteligencia emocional.

\section{KEY WORDS}

Competences; Socio-emotional competences; Competences Based Teacher Training; Emotional intelligence.

\section{PERFIL ACADÉMICO Y PROFESIONAL DE LOS AUTORES}

María L. Pertegal-Felices, licenciada en Psicopedagogía y doctoranda en el Departamento de Psicología Evolutiva y Didáctica de la Universidad de Alicante (España). Actualmente desarrolla su labor profesional como Profesora de educación primaria y como Colaboradora honorífica de la Universidad de Alicante. Sus líneas de investigación incluyen la inteligencia emocional aplicada al rendimiento en el trabajo, la investigación en educación superior y el desarrollo de las competencias genéricas.

Juan L. Castejón-Costa, catedrático de Psicología de la Educación y Director del Departamento de Psicología Evolutiva y Didáctica de la Universidad de Alicante (España). Está doctorado por la Universidad de Valencia (España). Ha sido Investigador Asociado en la Universidad de Yale (EEUU). Sus líneas de investigación incluyen la evaluación escolar, la motivación y la inteligencia emocional en el ámbito laboral.

María Ángeles Martínez Ruiz, catedrática del área de Didáctica y Organización Escolar, así como en la actualidad Decana de la Facultad de Educación de la Universidad de Alicante. Ha sido además Directora del Instituto de Ciencias de la Educación de la misma Universidad, donde impulsó la creación de Redes de investigación universitaria. Sus líneas de investigación se centran en el estudio de la educación superior y el desarrollo profesional docente en distintos niveles educativos.

\author{
Dirección de los autores: María Ángeles Martínez Ruiz \\ Departamento de Didáctica General y Di- \\ dácticas Específicas \\ Facultad de Educación \\ Universidad de Alicante \\ Carretera de San Vicente s/n \\ 03007 Alicante.
}


Fecha Recepción del Artículo: 24. marzo. 2010

Fecha Revisión del Artículo: 21. junio. 2010

Fecha Aceptación del Artículo: 29. septiembre. 2010 